\title{
Approximate solutions of fuzzy differential equations of fractional order using modified reproducing kernel Hilbert space method
}

\author{
Asia Khalaf Albzeirat ${ }^{a, *}$, Muhammad Zaini Ahmad ${ }^{a}$, Shaher Momani ${ }^{b, c}$, Norazrizal Aswad Abdul Rahman ${ }^{a}$ \\ a Institute of Engineering Mathematics, Universiti Malaysia Perlis, Pauh Putra Main Campus, 02600 Arau, Perlis, Malaysia. \\ ${ }^{b}$ Department of Mathematics, Faculty of Science, The University of Jordan, Amman 11942, Jordan. \\ ${ }^{c}$ Nonlinear Analysis and Applied Mathematics (NAAM) Research Group, Faculty of Science, King Abdulaziz University, Jeddah 21589, \\ Saudi Arabia.
}

Communicated by W. Shatanawi

\begin{abstract}
In this paper, we use the modified reproducing kernel Hilbert space method to approximate the solution of fuzzy differential equations of fractional order. Using this method, we construct a new algorithm to approximate the solution of such differential equations. The proposed algorithm produces solutions in terms of interval-valued fuzzy numbers. Two numerical examples are tested and the results showed that the proposed algorithm is able to produce solutions that approach to the exact solutions. It concludes that the proposed algorithm can be considered as a modern algorithm that complements to the existing ones. (C) 2017 All rights reserved.
\end{abstract}

Keywords: Caputo fractional derivative, fuzzy differential equation of fractional order, modified reproducing kernel Hilbert space method.

2010 MSC: 34A08, 34A07, 47B32.

\section{Introduction}

For the last decade, fuzzy differential equations (FDEs) have been studied by many researchers in different approaches $[3,12,29,31,35]$. All of these studies aimed to find the solutions of fuzzy differential equations of fractional order. This involves the process of introducing new methods as well as improving the classical methods to solve such differential equations. It is a fact that the improvement of problem solving tools effects directly how we handle the world around us. For instance, in [9] the authors proposed a new algorithm to find multiple solutions of the fractional differential equations. Thus, a further research has to be made to construct new methods, or by improving the existing classical methods direct or indirectly $[33,34]$.

This paper considers the general form of fuzzy differential equation of fractional order possessing Caputo fractional derivative:

$$
\begin{aligned}
D_{c, a, z}^{\alpha} M(z) & =g(z, M(z)), \\
M\left(z_{0}\right) & =z_{F}=(A, B, C),
\end{aligned}
$$

\footnotetext{
*Corresponding author

Email address: asiajor@yahoo.com (Asia Khalaf Albzeirat)
} 
where $D_{c, a, z}^{\alpha} M(z)$ is the fractional derivative in the sense of Caputo, $0<\alpha \leqslant 1, z>z_{0}$ and $M\left(z_{0}\right)=$ $(A, B, C)$ is a fuzzy initial value.

At instance, fuzzy differential equations of fractional order might be very complicated to be solved. Unlike ordinary differential equations, there are a few methods that have been proposed for solving fuzzy differential equations. Many of them are extension from the classical methods (see in $[5,17,19,24,38]$ ). There are several methods proposed by the authors to solve Eq. (1.1) (see in $[4,6-8,26])$.

From the previous studies, it was shown that the reproducing kernel theory (RKT) is popular in solving ordinary differential equations. The RKT was first developed in 1908 by Zaremba [14]. Since then, many modifications have been made. For example, Higgins [22] provided the crucial modification for this method. Furthermore, Mercer [30] improved this theory and demonstrated it on several real life applications.

In the last decades, the RKT has been used by many researchers in solving problems in signal processing, estimation theory, stochastic process, and hybrid wavelet (see in [10, 21, 23, 36]). Currently, some researchers expanded the application of RKT for finding the solution of fractional problems [1, 2, 11, 28, 37]. From these studies, it is necessary to focus on the application of this method in solving fuzzy differential equation of fractional order. Therefore, in this study we propose a new procedure on solving fuzzy differential equation of fractional order based on RKT.

The rest of this paper is organized as follows. In Section 2, we provide basic concepts of fractional calculus, fuzzy set and the RKT. In Section 3, we propose a new algorithm for approximating the solution of fuzzy differential equation of fractional order. While in Section 4, we present two numerical examples to show the effectiveness of the proposed algorithm. Finally, conclusion will be drawn in the last section.

\section{Basic concepts}

This section briefly elaborates the basic concepts of fractional calculus, fuzzy differential equations of fractional order, and reproducing kernel Hilbert space.

Definition 2.1 ([20]). The left fractional integral and the right fractional integral in the sense of RiemannLiouville for $\alpha \in(0,1)$ are defined as

$$
\mathrm{J}_{\mathrm{RL}, \mathrm{a}, z}^{\alpha} \mathrm{M}(z)=\frac{1}{\Gamma(\alpha)} \int_{\mathrm{a}}^{z}(z-\zeta)^{\alpha-1} \mathrm{M}(\zeta) \mathrm{d} \zeta,
$$

and

$$
\mathrm{J}_{\mathrm{RL}, z, \mathrm{~b}}^{\alpha} \mathrm{M}(z)=\frac{1}{\Gamma(\alpha)} \int_{z}^{\mathrm{b}}(\zeta-z)^{\alpha-1} \mathrm{M}(\zeta) \mathrm{d} \zeta,
$$

respectively.

Definition 2.2 ([32]). The left Caputo fractional derivative is defined as follows.

$$
\mathrm{D}_{\mathrm{c}, \mathrm{a}, z}^{\alpha} \mathrm{M}(z)=\frac{1}{\Gamma(\lceil\alpha\rceil-\alpha)} \int_{\alpha}^{z}(z-\zeta)^{\lceil\alpha\rceil-\alpha-1} \mathrm{M}^{\lceil\alpha\rceil}(\zeta) \mathrm{d} \zeta,
$$

where $M^{\lceil\alpha\rceil}=\frac{\mathrm{dM}^{\lceil\alpha\rceil}(\zeta)}{\mathrm{d} \zeta[\alpha]}$ and $\lfloor\alpha\rfloor \leqslant \alpha<\lceil\alpha\rceil, \alpha \in \mathrm{Z}^{+}$, where the symbol $\lceil\cdot\rceil$ denotes the nearest integer number greater than $\alpha$ and $\lfloor\cdot\rfloor$ denotes the nearest integer number less than $\alpha$. While the right Caputo fractional derivative is defined as

$$
\mathrm{D}_{\mathrm{c}, \mathrm{b}, z}^{\alpha} \mathrm{M}(z)=\frac{(-1)^{\lceil\alpha\rceil}}{\Gamma(\lceil\alpha\rceil-\alpha)} \int_{z}^{\mathrm{b}}(\zeta-z)^{\lceil\alpha\rceil-\alpha-1} \mathrm{M}^{\lceil\alpha\rceil}(\zeta) \mathrm{d} \zeta .
$$

The properties of the Caputo fractional derivative [27] is listed below.

(i) $D_{c, a, z}^{\alpha} J_{R L, a, z}^{\alpha} M(z)=M(z)$; 
(ii) $J_{R L, a, z}^{\alpha} D_{c, a, z}^{\alpha} M(z)=M(z)-\sum_{k=0}^{\lfloor\alpha\rfloor}\left(M^{(k)}(0) \cdot \frac{Z^{k}}{k !}\right), z>0,\lfloor\alpha\rfloor \leqslant \alpha<\lceil\alpha\rceil \in Z^{+}$.

We denote the set of all real numbers by $\mathbb{R}$ and the space of $n$-dimensional fuzzy numbers by $\mathbb{R}_{\mathrm{F}}^{n}$ where $\mathfrak{u}_{\mathrm{F}}(z): \mathbb{R}^{\mathfrak{n}} \rightarrow[0,1]$.

Definition 2.3. Let a fuzzy number $\mathfrak{u}_{\mathrm{F}}(z) \in \mathbb{R}_{\mathrm{F}}$ and $r \in[0,1]$. The $r$-cut of $\mathfrak{u}_{\mathrm{F}}(z)$ is the crisp set that contains all elements with degree in $\mathfrak{u}_{\mathrm{F}}(z)$ either greater than or equal to $r$ such that

$$
\left[u_{\mathrm{F}}(z)\right]^{\mathrm{r}}=\left\{z \in \mathbb{R}: u_{\mathrm{F}}(z) \geqslant \mathrm{r}\right\} .
$$

For fuzzy number $\mathfrak{u}_{\mathrm{F}}(z)$, its $r$-cut are closed and bounded interval in $\mathbb{R}$ and we denoted them by

$$
\left[\mathfrak{u}_{\mathrm{F}}(z)\right]^{\mathrm{r}}=\left[\mathrm{u}_{1,1 \mathrm{r}}(z), \mathrm{u}_{1,2 \mathrm{r}}(z)\right],
$$

where $u_{1,1 \mathrm{r}}(z)=\min \left\{z \mid z \in\left[\mathfrak{u}_{\mathrm{F}}(z)\right]^{\mathrm{r}}\right\}$ and $u_{1,2 \mathrm{r}}(z)=\max \left\{z \mid z \in\left[\mathfrak{u}_{\mathrm{F}}(z)\right]^{\mathrm{r}}\right\}$ for every $r \in[0,1]$.

For more details on r-cut of fuzzy numbers, please see in $[13,15,16,25,39]$. Fuzzy numbers are often classified into membership function. One of the most commonly used membership functions is the triangular membership function, or often referred as triangular fuzzy number.

Definition $2.4([4])$. A fuzzy number $u_{\mathrm{F}}$ is called triangular fuzzy number if its membership function has the following form:

$$
u_{F}(z)= \begin{cases}0, & \text { if } z<A \\ \frac{z-A}{B-A}, & \text { if } A \leqslant z<B \\ \frac{C-z}{C-B}, & \text { if } B \leqslant z \leqslant C \\ 0, & \text { if } z>C\end{cases}
$$

and its $r$-cut is computed as follows:

$$
[u]^{r}=[A+r(B-A), C-r(C-B)], r \in[0,1] .
$$

Lemma 2.5. Let $\mathrm{M}(z)$ be a fuzzy-valued function such that $\mathrm{M}(z):[\mathrm{a}, \mathrm{b}] \rightarrow \mathbb{R}_{\mathrm{F}}$ where $\mathrm{M}(z)$ is a bounded and continuous function in open interval $(a, b)$. The fractional derivative of $\mathrm{M}_{\mathrm{F}}(z)$ in the sense of Caputo can be expressed using r-cut representation as follows:

$$
\left[D_{c, a, z}^{\alpha} M(z)\right]^{r}=\left[D_{c, a, z}^{\alpha} M(z)\right]^{r}=\left[D_{c, a, z}^{\alpha} M_{1,1 r}(z), D_{c, a, z}^{\alpha} M_{1,2 r}(z)\right]
$$

where $\mathrm{D}_{\mathrm{c}}^{\alpha}$ is the operation that its effective representation is limited to $\mathrm{M}_{\mathrm{F}}(z)$.

By using Definition 2.3 and the properties of Caputo fractional derivative, we have

$$
\begin{aligned}
{\left[J_{R L, a, z}^{\alpha} D_{c, a, z}^{\alpha} M(z)\right]^{r} } & =\left[J_{R L, a, z}^{\alpha} D_{c, a, z}^{\alpha} M_{1,1 r}(z), J_{R L, a, z}^{\alpha} D_{c, a, z}^{\alpha} M_{1,2 r}(z)\right] \\
& =\left[M_{1,1 r}(z)-\sum_{k=0}^{m-1} M_{1,1 r}^{k}\left(0^{+}\right) \frac{z^{k}}{k !}, M_{1,2 r}(z)-\sum_{k=0}^{m-1} M_{1,2 r}^{k}\left(0^{+}\right) \frac{z^{k}}{k !}\right] .
\end{aligned}
$$

Definition 2.6 ([18]). Let HS be a Hilbert space. Suppose that $\mathrm{U}_{\mathrm{y}}(z) \in \mathrm{HS}$ and $M(\mathrm{y}) \in \mathrm{HS}$ satisfy the following condition:

$$
\left\langle M(z), u_{y}(z)\right\rangle=M(y), \forall y \in Z,
$$

then,

(i) $\mathrm{U}_{\mathrm{y}}(z)$ is a reproducing kernel of $\mathrm{HS}$;

(ii) HS is a reproducing kernel space. 
Definition 2.7 ([18]). The function space $\mathrm{FS}_{2}^{\mathrm{m}}[\mathrm{a}, \mathrm{b}]$ is defined as

$$
\mathrm{FS}_{2}^{\mathfrak{m}}[\mathrm{a}, \mathrm{b}]=\left\{\mathrm{u} \mid \mathrm{u}^{(\mathfrak{i})} \text { is absolutely continuous, } \mathrm{i}=1, \ldots, \mathrm{m}-1, \mathrm{u}^{(\mathrm{m})} \in \mathrm{L}^{2}[\mathrm{a}, \mathrm{b}]\right\} .
$$

The inner product in $\mathrm{FS}_{2}^{\mathrm{m}}[\mathrm{a}, \mathrm{b}]$, for any functions $u(z), v(z) \in \mathrm{FS}_{2}^{\mathrm{m}}[\mathrm{a}, \mathrm{b}]$ is defined as follows:

$$
\langle u, v\rangle_{F_{2}^{m}[a, b]}=\sum_{i=0}^{m-1} u^{i}(a) v^{i}(a)+\int_{a}^{b} u^{(m)} v^{(m)} d z .
$$

While the norm in $\mathrm{FS}_{2}^{\mathrm{m}}[\mathrm{a}, \mathrm{b}]$ for any functions $\mathrm{u}(z), v(z) \in \mathrm{FS}_{2}^{\mathrm{m}}[\mathrm{a}, \mathrm{b}]$ is defined as follows:

$$
\|u\|_{F S_{2}^{m}[a, b]}=\sqrt{\langle u, u\rangle_{F S_{2}^{m}[a, b]}}
$$

Definition 2.8 ([18]). The inner product space of the function space $\mathrm{FS}_{2}^{1}[\mathrm{a}, \mathrm{b}]$ is defined as

$$
\langle u, v\rangle_{F_{2}^{1}[a, b]}=u(0) v(0)+\int_{a}^{b} u^{(1)}(z) v^{(1)}(z) d z, \forall u, v \in F_{2}^{1}[a, b],
$$

and the norm of $\mathrm{FS}_{2}^{1}[\mathrm{a}, \mathrm{b}]$ is defined as

$$
\|\mathrm{u}\|_{\mathrm{FS}_{2}^{1}[\mathrm{a}, \mathrm{b}]}=\sqrt{\langle\mathrm{u}(z), \mathrm{u}(z)\rangle_{\mathrm{FS}_{2}^{1}[\mathrm{a}, \mathrm{b}]}} .
$$

Theorem 2.9 ([18]). The function space $\mathrm{FS}_{2}^{1}[\mathrm{a}, \mathrm{b}]$ is a reproducing kernel Hilbert space with reproducing kernel given by

$$
\mathrm{U}_{z}(\mathrm{y})= \begin{cases}1+y, & \text { if } y \leqslant z \\ 1+z, & \text { if } y>z\end{cases}
$$

Definition 2.10 ([18]). The inner product space of the function space $\mathrm{FS}_{2}^{2}[\mathrm{a}, \mathrm{b}]$ is defined as follows:

$$
\langle u, v\rangle_{F_{2}^{2}[a, b]}=\sum_{i=0}^{1} u^{(i)}(a) v^{(i)}(a)+\int_{a}^{b} u^{(2)}(z) v^{(2)}(z) d z, \quad \forall u, v \in \operatorname{FS}_{2}^{2}[a, b],
$$

and the norm of $F S_{2}^{1}[a, b]$ is defined as:

$$
\|\mathrm{u}\|_{\mathrm{FS}_{2}^{2}[\mathrm{a}, \mathrm{b}]}=\sqrt{\langle\mathrm{u}(z), \mathrm{u}(z)\rangle_{\mathrm{FS}_{2}^{2}[\mathrm{a}, \mathrm{b}]}} .
$$

Theorem 2.11 ([18]). The function space $\mathrm{FS}_{2}^{2}[\mathrm{a}, \mathrm{b}]$ is a reproducing kernel Hilbert space with reproducing kernel given by

$$
K_{z}(y)= \begin{cases}1+z y+\frac{z y^{2}}{2}-\frac{y^{3}}{6}, & \text { if } y \leqslant z \\ 1+z y+\frac{z^{2} y}{2}-\frac{z^{3}}{6}, & \text { if } y>z\end{cases}
$$

\section{New algorithm for fuzzy differential equations of fractional order}

In order to use reproducing kernal theory (RKT) as the tool, we need to homogenize the initial condition in Eq. (1.1). For that, we consider

$$
\begin{aligned}
M^{*}(z) & =M(z)-z_{F}, M^{*}\left(z_{0}\right)=0, \\
M(z) & =M^{*}(z)+z_{F} .
\end{aligned}
$$


After the homogenization, Eq. (1.1) can be converted to the following form:

$$
\left\{\begin{array}{l}
\left(\operatorname{LM}^{*}\right)(z)=g^{*}\left(z, M^{*}(z)\right), \\
M^{*}\left(z_{0}\right)=0 .
\end{array}\right.
$$

By applying the fuzzy set theory, Eq. (3.1) is converted into the following form:

$$
\begin{aligned}
\mathrm{D}_{\mathrm{c}, \mathrm{a}, z}^{\beta} M_{1,1 \mathrm{r}}^{*}(z) & =\mathrm{g}_{1,1 \mathrm{r}}^{*}\left(z, M_{1,1 \mathrm{r}}^{*}(z), \mathrm{M}_{1,2 \mathrm{r}}^{*}(z)\right), \\
\mathrm{D}_{\mathrm{c}, \mathrm{a}, z}^{\beta} M_{1,2 \mathrm{r}}^{*}(z) & =\mathrm{g}_{1,2 \mathrm{r}}^{*}\left(z, M_{1,1 \mathrm{r}}^{*}(z), \mathrm{M}_{1,2 \mathrm{r}}^{*}(z)\right), \\
\mathrm{M}_{1,1 \mathrm{r}}^{*}(z) & =0, M_{1,2 \mathrm{r}}^{*}(z)=0,
\end{aligned}
$$

and Eq. (3.2) is converted into the following form:

$$
\left\{\begin{array}{l}
\left(L_{1, j r} M_{1, j r}^{*}\right)(z)=g_{1, j r}^{*}\left(z, M_{1, j r}^{*}(z)\right), \\
M_{1, j r}^{*}\left(z_{0}\right)=0, \quad j=1,2 .
\end{array}\right.
$$

From [18], the reproducing kernel method is employed for solving Eq. (3.4). This includes three main steps that are also used to solve ordinary differential equations. From those steps, we propose the following procedures.

i) First, we construct reproducing kernels $\mathrm{FS}_{2}^{2}[\mathrm{a}, \mathrm{b}]$ and $\mathrm{FS}_{2}^{1}[\mathrm{a}, \mathrm{b}]$ for Eq. (3.4), after that we introduce a linear operator as follows:

$$
\mathrm{L}_{\mathrm{m}, \mathrm{jr}}=\mathrm{FS}_{2}^{2}[\mathrm{a}, \mathrm{b}] \rightarrow \mathrm{FS}_{2}^{1}[\mathrm{a}, \mathrm{b}], \quad \mathrm{m}=1, \quad \mathrm{j}=1,2 .
$$

Therefore, Eq. (3.3) is converted into the following form.

$$
\begin{aligned}
& M_{1,1 \mathrm{r}}^{*}(z)=\mathrm{L}_{1,1 \mathrm{r}}^{\mathrm{ad}} \mathrm{g}_{1,1 \mathrm{r}}^{*}\left(z, \mathrm{M}_{1,1 \mathrm{r}}^{*}(z), \mathrm{M}_{1,2 \mathrm{r}}^{*}(z)\right), \\
& \mathrm{M}_{1,2 \mathrm{r}}^{*}(z)=\mathrm{L}_{1,2 \mathrm{r}}^{\mathrm{ad}} \mathrm{g}_{1,2 \mathrm{r}}^{*}\left(z, M_{1,1 \mathrm{r}}^{*}(z), M_{1,2 \mathrm{r}}^{*}(z)\right),
\end{aligned}
$$

where $L_{1,1 r}^{a d}$ and $L_{1,2 r}^{a d}$ are adjoint operator of $L_{m, j r}, m=1$ and $j=1,2$.

ii) Then, we let $\left\{z_{k}\right\}_{k=1}^{\infty}$ to be a countable dense set in $[a, b]$ and let $e_{k, m, j r}(z)=k_{z_{k}}(y) . k_{z}(y)$ is the reproducing kernel of $\mathrm{FS}_{2}^{1}[\mathrm{a}, \mathrm{b}], \forall \mathrm{M}_{\mathrm{m}, \mathrm{jr}}^{*}(z) \in \mathrm{FS}_{2}^{1}[\mathrm{a}, \mathrm{b}], \mathrm{m}=1$ and $j=1$, 2. Hence,

$$
\left\langle M_{\mathrm{m}, \mathrm{jr}}^{*}(z), e_{\mathrm{k}, \mathrm{m}, \mathrm{jr}}(z)\right\rangle_{\mathrm{FS}_{2}^{1}}=\left\langle\mathrm{M}_{\mathrm{m}, \mathrm{jr}}^{*}(z), \mathrm{k}_{z}(\mathrm{y})\right\rangle_{\mathrm{FS}_{2}^{1}}=M_{\mathrm{m}, \mathrm{jr}}^{*}(z)
$$

Additionally, by allowing

$$
\mathrm{L}_{\mathrm{m}, \mathrm{jr}}^{\mathrm{ad}} e_{k, m, j r}(z)=\Psi_{k, m, j r}(z)
$$

we have

$$
\left\langle M_{\mathrm{m}, \mathrm{jr}}^{*}(z), \Psi_{k, \mathrm{~m}, \mathrm{jr}}(z)\right\rangle_{\mathrm{FS}}=\left\langle\mathrm{M}_{\mathrm{m}, \mathrm{jr}}^{*}(z), \mathrm{L}_{1,1 \mathrm{r}}^{\mathrm{ad}} e_{k, m, j r}(z)\right\rangle_{\mathrm{FS}}=\left\langle\mathrm{L}_{\mathrm{m}, \mathrm{jr}} \mathrm{M}_{\mathrm{m}, \mathrm{jr}}^{*}(z), e_{k, m, j r}(z)\right\rangle_{\mathrm{F} S_{2}^{1}} .
$$

Thus, $\Psi_{k, m, j r}(z)$ can be expressed as follows:

$$
\begin{aligned}
\Psi_{k, m, j r}(z)=\mathrm{L}_{m, j r}^{a d} e_{k, m, j r}(z) & =\left\langle\mathrm{L}_{m, j r}^{\mathrm{ad}} e_{k, m, j r}(z), K_{z}(y)\right\rangle_{F S_{2}^{2}[a, b]} \\
& =\left\langle e_{k, m, j r}(z), \mathrm{L}_{m, j r} K_{z}(y)\right\rangle_{F_{2}^{1}[a, b]}=\left.L_{y} K_{z}(y)\right|_{y=z_{k}} .
\end{aligned}
$$

From here, we obtain the following result.

Theorem 3.1. If $\left\{z_{\mathrm{k}}\right\}_{\mathrm{k}=1}^{\infty}$ is a dense set in $[\mathrm{a}, \mathrm{b}]$, then the complete system of the function space $\mathrm{FS}_{2}^{2}[\mathrm{a}, \mathrm{b}]$ under fuzzy setting is as follows:

$$
\left\{\Psi_{k, m, j r}(z)\right\}_{(k, m, j r)=(1,1,1 r)}^{(\infty, 1,2 r)} .
$$


Proof. For each fixed $M_{m, j r}^{*}(z) \in \mathrm{FS}_{2}^{2}[a, b]$, and let $\left\langle M_{m, j r}^{*}(z), \Psi_{k, m, j r}(z)\right\rangle=0, k=1, \ldots$, then

$$
\begin{aligned}
\left\langle M_{m, j r}^{*}(z), \Psi_{k, m, j r}(z)\right\rangle_{F_{2}^{2}[a, b]} & =\left\langle M_{m, j r}^{*}(z), L_{1,1 r}^{a d} e_{k, m, j r}(z)\right\rangle_{F_{2}^{2}[a, b]} \\
& =\left\langle L_{m, j r} M_{m, j r}^{*}(z), e_{k, m, j r}(z)\right\rangle_{F S_{2}^{1}[a, b]} \\
& =L_{m, j r} M_{m, j r}^{*}\left(z_{k}\right)=0 .
\end{aligned}
$$

Since $\left\{z_{k}\right\}_{k=1}^{\infty}$ is dense in $[a, b]$, therefore, $L_{m, j r} M_{m, j r}^{*}\left(z_{k}\right)=0, \forall m=1$ and $j=1,2$. It follows that $M_{m, j r}^{*}(z)=0$ from the existence of the inverse operator of $L_{m, j r}$ and the continuity of $M_{m, j r}^{*}(z)$.

iii) For the third step, we construct an orthonormal system $\left\{\bar{\Psi}_{k, m, j r}\right\}_{(k, m, j r)=(1,1,1 r)}^{(\infty, 1,2 r)}$ of $F S_{2}^{2}[a, b]$ by using Gram-Schmidt orthogonalization process.

$$
\bar{\Psi}_{k, m, j r}(z)=\sum_{l=1}^{k} \beta_{k l, m, j r}(z) \Psi_{k, m, j r}(z), \forall m=1, j=1,2, l=1,2, \ldots, k,
$$

where $\beta_{\mathrm{kl}, \mathrm{m}, \mathrm{jr}}(z)$ is the orthogonalization coefficient.

From the third step we obtain the following results.

Theorem 3.2. Let $\left\{z_{k}\right\}_{k=1}^{\infty}$ be a dense set in $[a, b]$, and the solution of (3.3) is unique on $\mathrm{FS}_{2}^{1}[\mathrm{a}, \mathrm{b}]$. Then, the solution of (3.3) can be written as follows.

$$
M_{m, j r}^{*}(z)=\sum_{k=1}^{\infty} \sum_{l=1}^{k} \beta_{k l, m, j r} g_{m, j r}^{*}\left(z_{l}, M_{m, j r}^{*}\left(z_{l}\right) \bar{\Psi}_{k, m, j r}(z)\right), \forall m=1, j=1,2 .
$$

Proof. By applying Theorem 3.1, $\left\{\bar{\Psi}_{k, m, j r}\right\}_{(k, m, j r)=(1,1,1 r)}^{(\infty, 1,2 r)}$ is a complete orthonormal basis for $\mathrm{FS}_{2}^{2}[\mathrm{a}, \mathrm{b}]$. So, $M_{m, j r}^{*}(z)$ can be extended into Fourier series for orthonormal system $\left\{\bar{\Psi}_{k, m, j r}\right\}_{(k, m, j r)=(1,1,1 r)}^{(\infty, 1,2 r)}$ as follows:

$$
M_{m, j r}^{*}(z)=\sum_{k=1}^{\infty}\left\langle M_{m, j r}^{*}(z), \bar{\Psi}_{k, m, j r}\right\rangle \Psi_{k, m, j r}(z), \forall m=1, j=1,2,
$$

where $\mathrm{FS}_{2}^{2}[\mathrm{a}, \mathrm{b}]$ is a reproducing kernel Hilbert space. Therefore,

$$
M_{m, j r}^{*}(z)=\sum_{k=1}^{\infty}\left\langle M_{m, j r}^{*}(z), \bar{\Psi}_{k, m, j r}\right\rangle \Psi_{k, m, j r}(z), \forall m=1, j=1,2,
$$

converges in the sense of the norm in Definition 2.10. By Definition 2.6, we have

$$
\begin{aligned}
M_{m, j r}^{*}(z) & =\sum_{k=1}^{\infty}\left\langle M_{m, j r}^{*}(z), \bar{\Psi}_{k, m, j r}\right\rangle \bar{\Psi}_{k, m, j r} \\
& =\sum_{k=1}^{\infty}\left\langle M_{m, j r}^{*}(z), \sum_{l=1}^{k} \beta_{k l, m, j r} \Psi_{k, m, j r}(z)\right\rangle_{F S_{2}^{2}[a, b]} \bar{\Psi}_{k, m, j r} \\
& =\sum_{k=1}^{\infty} \sum_{l=1}^{k} \beta_{k l, m, j r}(z)\left\langle M_{m, j r}^{*}(z), \Psi_{k, m, j r}(z)\right\rangle_{F S_{2}^{2}[a, b]} \bar{\Psi}_{k, m, j r} \\
& =\sum_{k=1}^{\infty} \sum_{l=1}^{k} \beta_{k l, m, j r}(z)\left\langle M_{m, j r}^{*}(z), L_{m, j r}^{a d} e_{l, m, j r}(z)\right\rangle_{F S_{2}^{2}[a, b]} \bar{\Psi}_{k, m, j r}
\end{aligned}
$$




$$
\begin{aligned}
& =\sum_{k=1}^{\infty} \sum_{l=1}^{k} \beta_{k l, m, j r}(z)\left\langle L_{m, j r} M_{m, j r}^{*}(z), e_{l, m, j r}(z)\right\rangle_{F S_{2}^{1}[a, b]} \bar{\Psi}_{k, m, j r} \\
& =\sum_{k=1}^{\infty} \sum_{l=1}^{k} \beta_{k l, m, j r}(z)\left\langle g_{m, j r}^{*}\left(z, M_{m, j r}^{*}(z)\right), e_{l, m, j r}(z)\right\rangle_{F S_{2}^{1}[a, b]} \bar{\Psi}_{k, m, j r} \\
& =\sum_{k=1}^{\infty} \sum_{l=1}^{k} \beta_{k l, m, j r}(z) g_{m, j r}^{*}\left(z_{l}, M_{m, j r}^{*}\left(z_{l}\right)\right) \bar{\Psi}_{k, m, j r}, \forall m=1, j=1,2 .
\end{aligned}
$$

Based on Theorem 3.2, the approximate solution of $M_{m, j r}(z)$ for $N$-terms is

$$
M_{m, j r}^{* N}(z)=\sum_{k=1}^{N} \sum_{l=1}^{k} \beta_{k l, m, j r}(z) g_{m, j r}^{*}\left(z_{l}, M_{m, j r}^{*}\left(z_{l}\right)\right) \bar{\Psi}_{k, m, j r}, \forall m=1, j=1,2 .
$$

Now, by using Eq. (3.1), the new form of $M_{m, j r}^{N}(z)$ is as

$$
M_{m, j r}^{N}(z)=\left(\sum_{k=1}^{N} \sum_{l=1}^{k} \beta_{k l, m, j r}(z) g_{m, j r}\left(z_{l}, M_{m, j r}\left(z_{l}\right)\right) \bar{\Psi}_{k, m, j r}\right)+z_{F}, \forall m=1, j=1,2 .
$$

The third procedure is simplified in the following algorithm.

Algorithm 3.3. Procedure for approximating the solution of Eq. (3.1).

Input: In this part, we consider the interval $[a, b]=[0,1]$, the integer $N$, the kernel $k_{z}(y)$ and the differential operator $L_{y, 1, j r}$ from the definition for left Caputo fractional derivative. We also consider the function $g_{1, j r}^{*}\left(z, M_{1,1 \mathrm{r}}^{*}(z)\right)=g_{1, j r}\left(z, M_{1,1 \mathrm{r}}^{*}(z)+z_{\mathrm{F}, j r_{\mathrm{d}}}\right)$, the integer $m$, with fuzzy initial condition $z_{\mathrm{F}}=(\mathrm{a}, \mathrm{b}, \mathrm{c})$ and the value of $\alpha$.

First, we approximate the solution of $\mathrm{M}_{1, j \mathrm{r}}(z)$.

Step 1 : Let $k \in[0,1]$ and $y \in[0,1]$. Then,

$$
K_{z}(y)= \begin{cases}\left(1+z y+\frac{z y^{2}}{2}-\frac{y^{3}}{6}\right), & z \leqslant y \\ \left(1+z y+\frac{y z^{2}}{2}-\frac{z^{3}}{6}\right), & \text { otherwise. }\end{cases}
$$

Step 2 : For the values $j=1,2$, follow Steps 3-6.

Step 3 : For $k=1,2, \ldots, N, j=1,2, d=1,2, \ldots, m$, set the values of $z, r$ and $\psi_{k, 1, j r_{d}}(z)$ as follows:

1. Set $z_{0}=0$.

2. Set $z_{k}=z_{k-1}+\frac{1}{N}$.

3. Set $r_{0}=0$.

4. Set $r_{d}=r_{d-1}+\frac{d}{m}$,

5. - if $j=1$, set $z_{F, j r_{d}}=a+r_{d}(b-a)$,

- if $j=2$, set $z_{F, j r_{d}}=c-r_{d}(c-b)$.

6. Set $\psi_{k, 1, j r_{d}}(z)=\left.L_{y, 1, j r} K_{z}(y)\right|_{y=z_{k}}$.

So, we get the following output

$$
\left\{\psi_{\mathrm{k}, 1, j r_{\mathrm{d}}}(z)\right\}_{\left(\mathrm{k}, 1, j \mathrm{r}_{\mathrm{d}}\right)}^{\left(\mathrm{N}, 1,2 \mathrm{r}_{\mathrm{d}}\right)}
$$

Step 4 : For $k=2, \ldots, N, s=1,2, \ldots, k$, set the values of $\beta, R$ and $d$ as follows.

1. Set $\beta_{11}=\frac{1}{\left\|\psi_{1,1, j r_{d}}\right\|}$. 
2. Set $\beta_{\mathrm{kk}}=\frac{1}{\left\|\mathrm{R}_{\mathrm{k}, 1, j \mathrm{r}_{\mathrm{d}}}\right\|}$.

3. Set $\left.\beta_{k p, 1, j r_{d}}=\frac{-1}{\left\|R_{k, 1, j r}\right\|}\left(\sum_{s=p}^{k-1} d_{k s, 1, j r} \beta_{s p, 1, j r_{d}}\right)\right), p<k$.

4. Set $R_{k, 1, j r_{d}}=\sqrt{\left\|\psi_{1,1, j r}\right\|^{2}-\sum_{s=p}^{k-1}\left(d_{k s, 1, j r_{d}}\right)^{2}}$.

5. Then, $d_{k s, 1, j r}=\psi_{k, 1, j r}, \overline{\psi_{k, 1, j r_{d}}}$.

Then, we get the following output

\section{$\beta_{\mathrm{ks}, 1, j \mathrm{r}_{\mathrm{d}}}$.}

Step 5 : For $k=1,2, \ldots, N$, we have

$$
\overline{\psi_{k, 1, j r_{d}}(z)}=\sum_{s=1}^{k} \beta_{k s} \psi_{s, 1, j r_{d}}(z) .
$$

Step $6:$ For $k=1,2, \ldots, N$, we set the values for $M$ as follows.

1. Set $M_{1, j r_{d}}^{*}\left(z_{0}\right)=0$.

2. Set $M_{1, j r}^{*}(z)=\sum_{k=1}^{N} \sum_{s=1}^{k} \beta_{k s, 1, j r}(z) g_{1, j r}\left(z, M_{1,1 r}^{*}\left(z_{s}\right)+z_{F, j r_{d}}\right) \bar{\psi}_{k, 1, j r}$.

3. Set $M_{1, j r_{d}}(z)=M_{1, j r_{d}}^{*}(z)+\left(z_{F, j r_{d}}\right)$.

Output: Then, we get the following output.

$$
M_{1, j r_{d}}(z)=\left[M_{1,1 r_{d}}(z)+M_{1,2 r_{d}}(z)\right] .
$$

\section{Numerical examples}

In this section, we provide two numerical examples.

Example 4.1. Consider the following fuzzy differential equation of order $\alpha=1$.

$$
\mathrm{D}_{\mathrm{c}, \mathrm{a}, \mathrm{z}}^{\alpha} \mathrm{M}(z)=2 z^{2}+3 z-\mathrm{M}(z), M\left(z_{0}\right)=(1,2,3), \quad 0 \leqslant z \leqslant 1 .
$$

By applying fuzzy set theory, we obtain

$$
\begin{array}{ll}
D_{c, a, z}^{\alpha} M_{1,1 r}(z)=2 z^{2}+3 z-M_{1,1 r}(z), & M_{1,1 r}\left(z_{0}\right)=1+r, r \in[0,1], \\
D_{c, a, z}^{\alpha} M_{1,1 r}(z)=2 z^{2}+3 z-M_{1,2 r}(z), & M_{1,2 r}\left(z_{0}\right)=3-r, r \in[0,1] .
\end{array}
$$

To solve the equation, we used the proposed algorithm in previous section and we let $\mathrm{N}=127$ and we consider the interval $[0,1]$. Then, the approximate results obtained are presented in Tables 1-5. The graphical representation of the solutions can be seen in Figures 1-10. In those figures, the results obtained using our method are also compared with the exact solutions within the same interval and having the same fuzzy initial value.

For $r=0$, the exact solutions are

$$
M_{1,1 \mathrm{r}}(z)=1-2 z+z^{2}, M_{1,2 \mathrm{r}}(z)=e^{-z}\left(2+e^{z}-z e^{z}+2 e^{z} z^{2}\right) .
$$

For $r=0.5$, the exact solutions are

$$
M_{1,1 \mathrm{r}}(z)=2 e^{-z}\left(0.25+0.5 e^{z}-0.5 z e^{z}+z^{2} e^{z}\right), \quad M_{1,2 \mathrm{r}}(z)=2 e^{-z}\left(0.75+0.5 e^{z}-0.5 z e^{z}+z^{2} e^{z}\right) .
$$

For $r=1$, the exact solutions are

$$
M_{1,1 \mathrm{r}}(z)=M_{1,2 \mathrm{r}}(z)=e^{-z}\left(1+z e^{z}-2 z^{2} e^{z}\right) .
$$


Table 1: Numerical solution for Example 4.1.

\begin{tabular}{cccc}
\hline$z$ & $\begin{array}{c}\mathrm{M}_{1,1 \mathrm{r}}(z) \\
\mathrm{r}=0\end{array}$ & $\begin{array}{c}\hat{M}_{1,1 \mathrm{r}}(z) \\
\mathrm{r}=0\end{array}$ & Errors \\
\hline 0.0 & 1.00 & 1.00 & 0.00 \\
0.1 & 0.92000 & 0.920001122 & $1.122011299 \times 10^{-6}$ \\
0.2 & 0.88000 & 0.8800022993 & $2.299334099 \times 10^{-6}$ \\
0.3 & 0.88000 & 0.8800034787 & $3.478728604 \times 10^{-6}$ \\
0.4 & 0.92000 & 0.9200046429 & $4.642932368 \times 10^{-6}$ \\
0.5 & 1.00000 & 1.000005923 & $4.642932368 \times 10^{-6}$ \\
0.6 & 1.12000 & 1.120007268 & $7.26794065 \times 10^{-6}$ \\
0.7 & 1.28000 & 1.280008596 & $8.595896458 \times 10^{-6}$ \\
0.8 & 1.48000 & 1.480010076 & $1.007556054 \times 10^{-5}$ \\
0.9 & 1.72000 & 1.720011705 & $1.007556054 \times 10^{-5}$ \\
1.0 & 2.00000 & 2.000013357 & $1.335720074 \times 10^{-5}$ \\
\hline
\end{tabular}

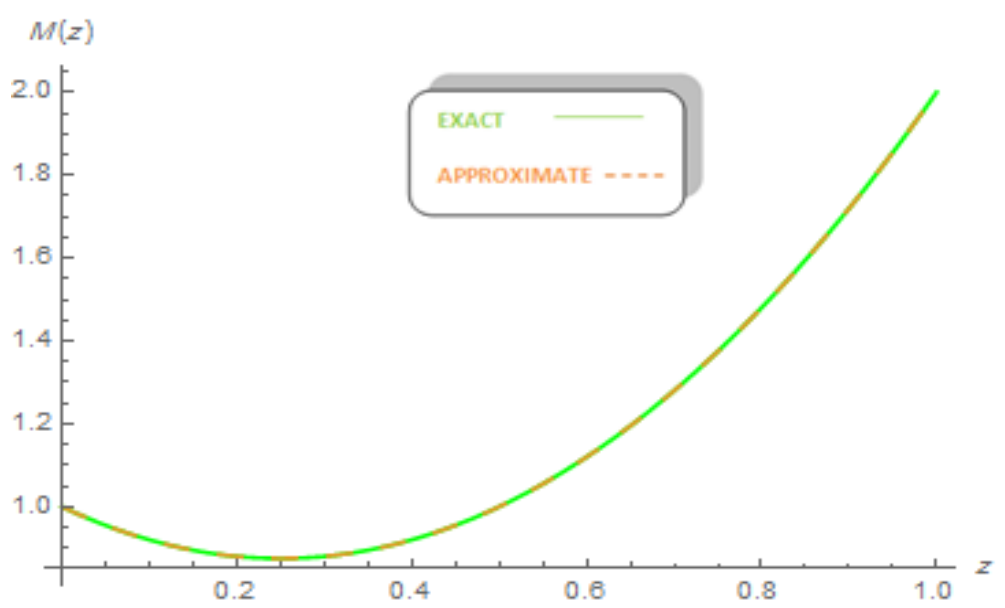

Figure 1: The exact and approximate solutions of $M_{1,1 r}(z)$, when $r=0$ for Example 4.1.

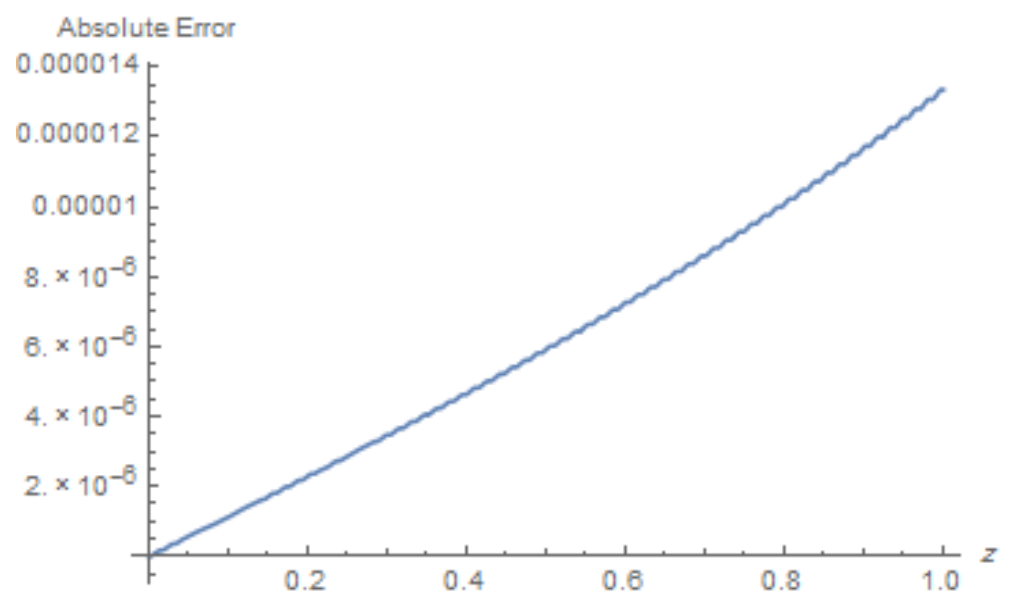

Figure 2: The errors in solutions for $M_{1,1 \mathrm{r}}(z)$, when $r=0$ for Example 4.1. 
Table 2: Numerical solutions of $M_{1,2 r}(z)$ when $r=0$ for Example 4.1.

\begin{tabular}{cccc}
\hline$z$ & $\begin{array}{c}M_{1,2 \mathrm{r}}(z) \\
\mathrm{r}=0\end{array}$ & $\begin{array}{c}\hat{M}_{1,2 \mathrm{r}}(z) \\
\mathrm{r}=0\end{array}$ & Errors \\
\hline 0.0 & 3.00 & 3.00 & 0.00 \\
0.1 & 2.729674836071919 & 2.729674122 & $7.143396936 \times 10^{-7}$ \\
0.2 & 2.5174615061559633 & 2.517460432 & $1.073837352 \times 10^{-6}$ \\
0.3 & 2.361636441363436 & 2.361635354 & $1.087723213 \times 10^{-6}$ \\
0.4 & 2.2606400920712786 & 2.260639284 & $8.082239611 \times 10^{-6}$ \\
0.5 & 2.213061319425267 & 2.213061076 & $2.436512458 \times 10^{-6}$ \\
0.6 & 2.2176232721880527 & 2.217623855 & $5.824507046 \times 10^{-6}$ \\
0.7 & 2.273170607582819 & 2.273172208 & $1.600046236 \times 10^{-6}$ \\
0.8 & 2.378657928234443 & 2.378660805 & $2.876281287 \times 10^{-6}$ \\
0.9 & 2.5331393194811986 & 2.533143726 & $4.406986805 \times 10^{-6}$ \\
1.0 & 2.7357588823428842 & 2.735764967 & $6.084919464 \times 10^{-6}$ \\
\hline
\end{tabular}

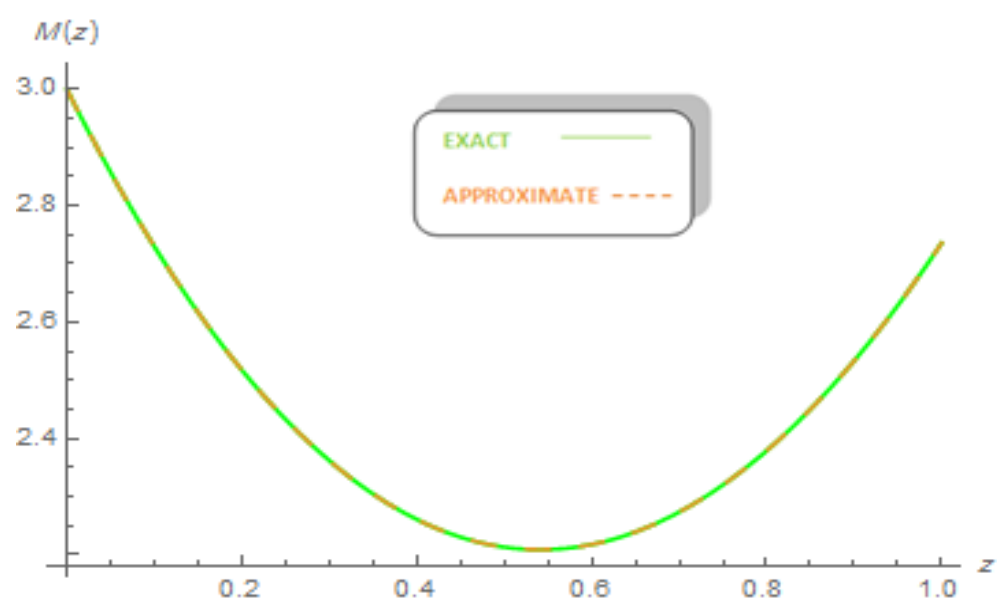

Figure 3: The exact and approximate solutions for $M_{1,2 r}(z)$ when $r=0$ for Example 4.1.

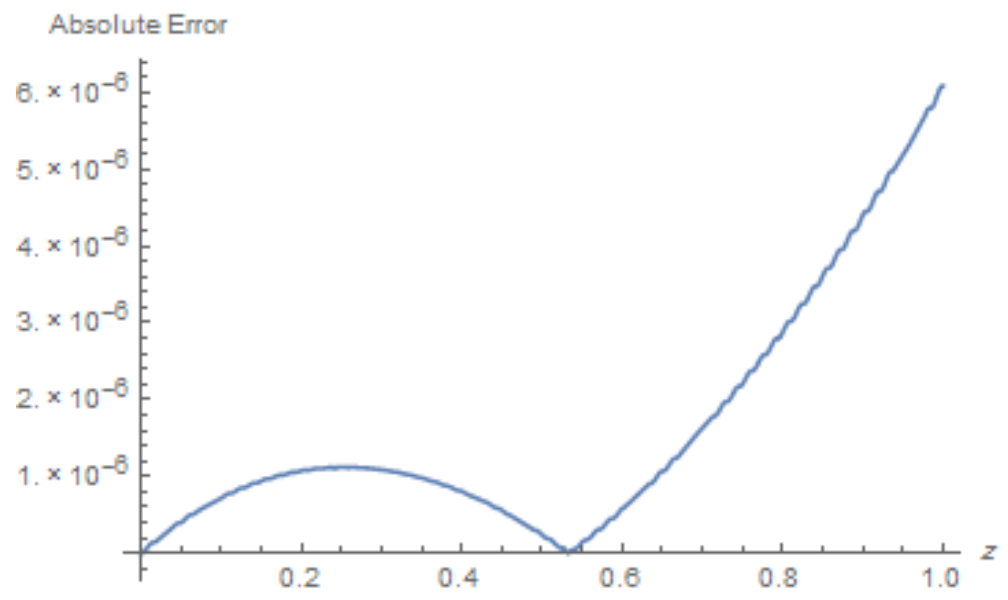

Figure 4: The errors in solutions for $M_{1,2 r}(z)$ when $r=0$ for Example 4.1. 
Table 3: Numerical solutions of $M_{1,2 r}(z)$ when $r=0$ for Example 4.1.

\begin{tabular}{cccc}
\hline$z$ & $\begin{array}{c}M_{1,1 \mathrm{r}}(z) \\
\mathrm{r}=0.5\end{array}$ & $\begin{array}{c}\hat{M}_{1,1 \mathrm{r}}(z) \\
\mathrm{r}=0.5\end{array}$ & Errors \\
\hline 0.0 & 1.50 & 1.50 & 0.00 \\
0.1 & 1.3724187090179798 & 1.372419372 & $6.629235505 \times 10^{-7}$ \\
0.2 & 1.2893653765389907 & 1.289366833 & $1.456041236 \times 10^{-6}$ \\
0.3 & 1.250409110340859 & 1.250411447 & $2.337115649 \times 10^{-6}$ \\
0.4 & 1.2551600230178197 & 1.255163303 & $3.280143285 \times 10^{-6}$ \\
0.5 & 1.3032653298563168 & 1.303269711 & $4.381550751 \times 10^{-6}$ \\
0.6 & 1.3944058180470131 & 1.394411415 & $5.596568163 \times 10^{-6}$ \\
0.7 & 1.7046644820586112 & 1.528299499 & $6.846933903 \times 10^{-6}$ \\
0.8 & 1.9232848298702998 & 1.704672758 & $8.275740723 \times 10^{-6}$ \\
0.9 & 1.9232848298702998 & 1.92329471 & $9.880615046 \times 10^{-6}$ \\
1.0 & 2.183939720585721 & 2.18395126 & $1.153913042 \times 10^{-5}$ \\
\hline
\end{tabular}

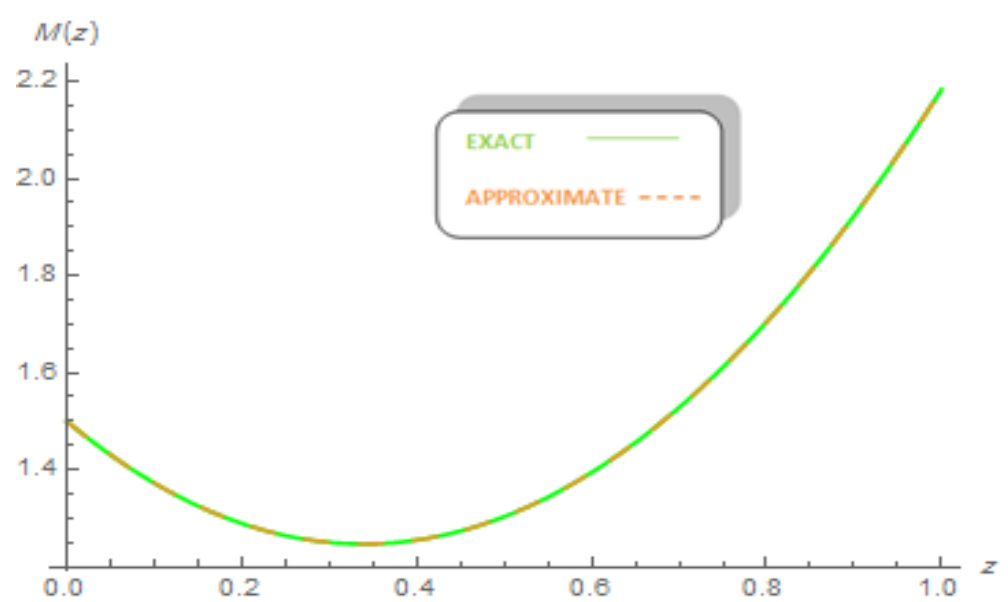

Figure 5: The exact and approximate of $M_{1,1 r}(z)$ when $r=0.5$ for Example 4.1.

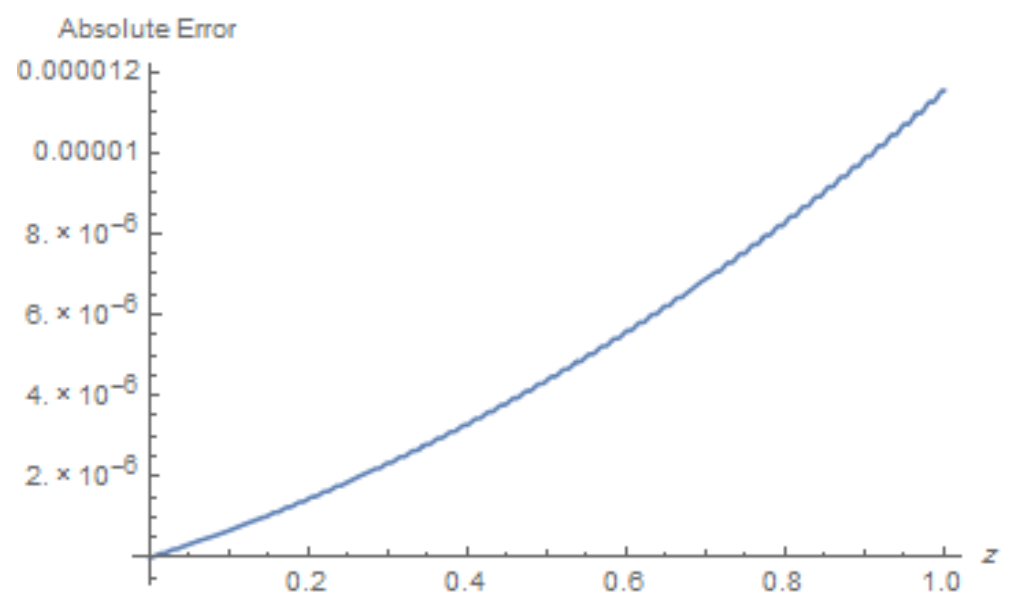

Figure 6: The errors in solutions for $M_{1,1 \mathrm{r}}(z)$ when $r=0.5$ for Example 4.1. 
Table 4: Numerical solutions of $M_{1,2 r}(z)$ when $r=0$ for Example 4.1.

\begin{tabular}{cccc}
\hline$z$ & $\begin{array}{c}\text { Exact solution } M_{1,2 \mathrm{r}}(z) \\
\mathrm{r}=0.5\end{array}$ & $\begin{array}{c}\hat{M}_{1,2 \mathrm{r}}(z) \\
\mathrm{r}=0.5\end{array}$ & Errors \\
\hline 0.0 & 2.50 & 2.50 & 0.00 \\
0.1 & 2.2772561270539393 & 2.277255872 & $2.5525194585 \times 10^{-7}$ \\
0.2 & 2.1080961296169725 & 2.108095899 & $2.305444893 \times 10^{-6}$ \\
0.3 & 1.991227331022577 & 1.991227385 & $5.388974111 \times 10^{-6}$ \\
0.4 & 1.925480069053459 & 1.925480624 & $5.545651214 \times 10^{-6}$ \\
0.5 & 1.9097959895689502 & 1.909797288 & $1.298082753 \times 10^{-6}$ \\
0.6 & 1.9432174541410394 & 1.943219708 & $2.253823192 \times 10^{-6}$ \\
0.7 & 2.0248779556871144 & 2.024881305 & $3.349008792 \times 10^{-6}$ \\
0.8 & 2.1539934461758325 & 2.153998122 & $4.6761011 \times 10^{-6}$ \\
0.9 & 2.329854489610899 & 2.329860721 & $6.231529552 \times 10^{-6}$ \\
1.0 & 2.5518191617571633 & 2.551827065 & $7.902989782 \times 10^{-5}$ \\
\hline
\end{tabular}

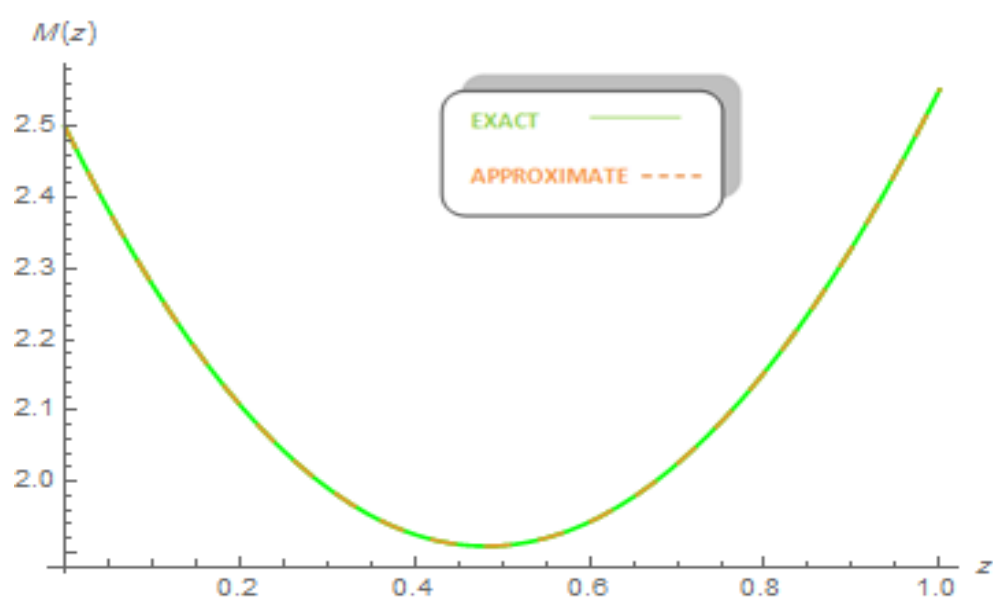

Figure 7: The exact and approximate solutions of $M_{1,2 r}(z)$ when $r=0.5$ for Example 4.1.

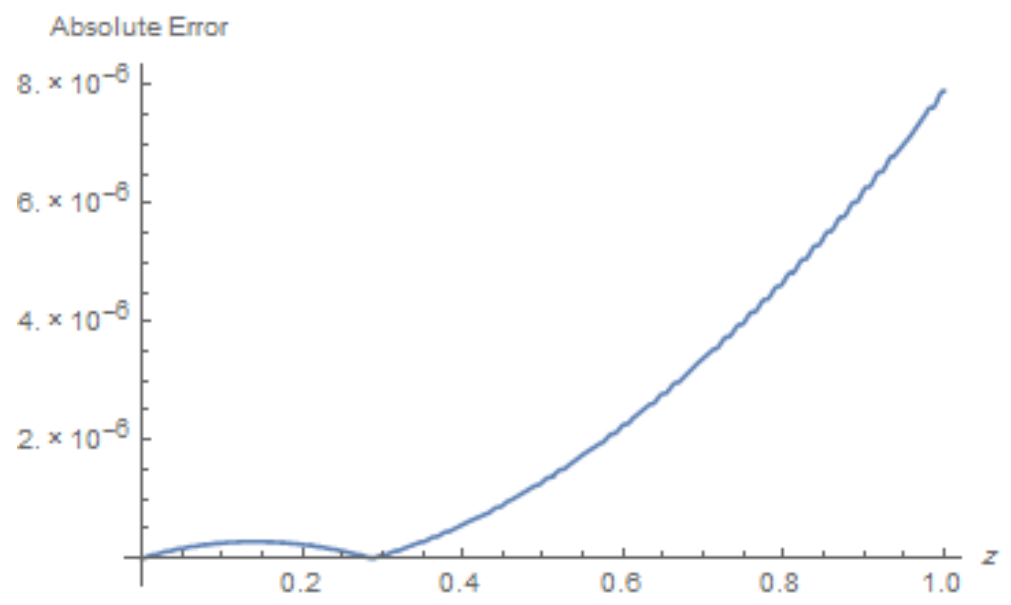

Figure 8: The errors in solutions for $M_{1,2 r}(z)$ when $r=0.5$ for Example 4.1. 
Table 5: Numerical solutions of $M_{1,1 \mathrm{r}}(z)$ and $M_{1,2 \mathrm{r}}(z)$ for different values of $\mathrm{r}$ and $\alpha=0.8$.

\begin{tabular}{cccc}
\hline$z$ & $M_{1,1 \mathrm{r}}(z)=\mathrm{M}_{1,2 \mathrm{r}}(z), \mathrm{r}=1.0$ & $\hat{M}_{1,1 \mathrm{r}}(z)=\mathrm{M}_{1,2 \mathrm{r}}(z), \mathrm{r}=1.0$ & Errors \\
& $\mathrm{r}=1.0$ & 2.00 & \\
\hline 0.0 & 2.00 & 1.824837622 & $2.038358025 \times 10^{-7}$ \\
0.1 & 1.8248374180359594 & 1.698731366 & $6.127483738 \times 10^{-7}$ \\
0.2 & 1.6987307530779816 & 1.620819416 & $1.195502695 \times 10^{-6}$ \\
0.3 & 1.620818220681718 & 1.590321963 & $1.917354203 \times 10^{-6}$ \\
0.4 & 1.5903200460356395 & 1.6065335 & $2.839816752 \times 10^{-6}$ \\
0.5 & 1.6065306597126334 & 1.668815561 & $3.925195678 \times 10^{-6}$ \\
0.6 & 1.6688116360940264 & 1.776590402 & $5.097971347 \times 10^{-6}$ \\
0.7 & 1.7765853037914097 & 1.92933544 & $6.475920912 \times 10^{-6}$ \\
0.8 & 1.929328964117222 & 2.126577716 & $8.05607230 \times 10^{-6}$ \\
0.9 & 2.1265696597405994 & 2.367889162 & $9.72106010 \times 10^{-5}$ \\
1.0 & 2.3678794411714423 & &
\end{tabular}

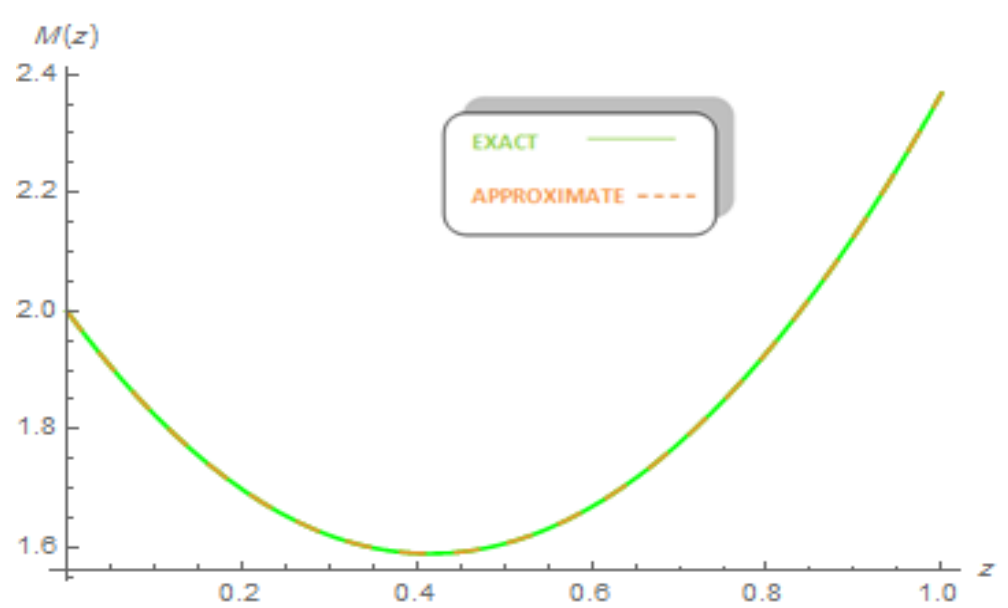

Figure 9: The exact and approximate solutions of $M_{1,1}(z)=M_{1,2 r}(z)$ when $r=1.0$ for Example 4.1.

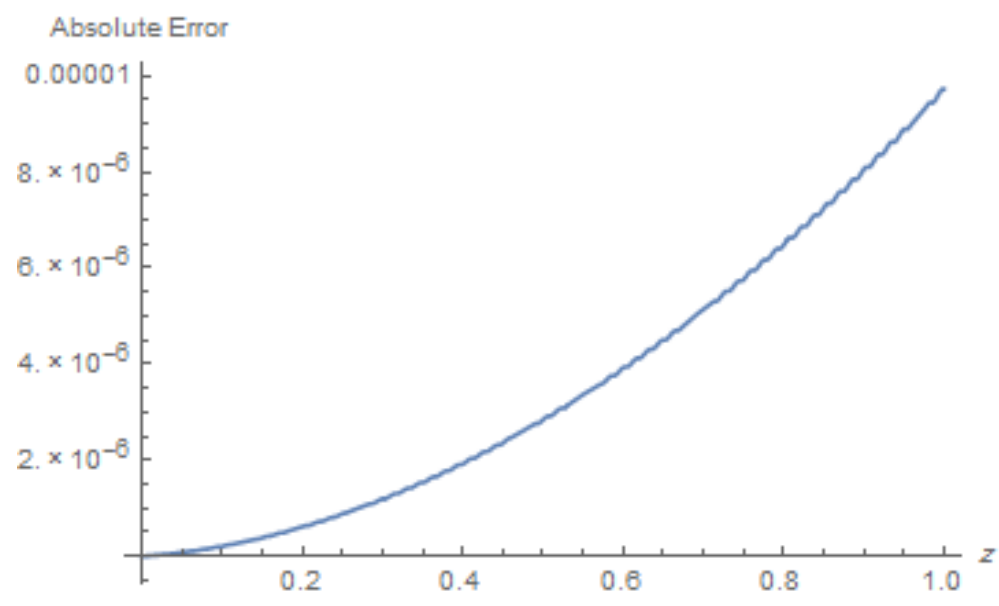

Figure 10: The errors in solutions for $M_{1,1 r}(z)=M_{1,2 r}(z)$ when $r=1.0$ for Example 4.1. 
Example 4.2. Consider the following fuzzy differential equation of order $\alpha=0.8$.

$$
\mathrm{D}_{\mathrm{c}, \mathrm{a}, z}^{\alpha} \mathrm{M}(z)=2 z^{2}+3 z-M(z), \quad M\left(z_{0}\right)=(2,3,4), 0 \leqslant z \leqslant 1 .
$$

By applying fuzzy set theory, we obtain

$$
\begin{array}{ll}
D_{c, a}^{\alpha} M_{1,1 \mathrm{r}}(z)=2 z^{2}+3 z-M_{1,1 \mathrm{r}}(z), & M_{1,1 \mathrm{r}}\left(z_{0}\right)=2+\mathrm{r}, \mathrm{r} \in[0,1], \\
\mathrm{D}_{\mathrm{c}, \mathrm{a}}^{\alpha} M_{1,1 \mathrm{r}}(z)=2 z^{2}+3 z-M_{1,2 \mathrm{r}}(z), & M_{1,2 \mathrm{r}}\left(z_{0}\right)=4-\mathrm{r}, \mathrm{r} \in[0,1] .
\end{array}
$$

Table 6 shows the results for Example 4.2 at $\alpha=0.8$ with the values of $r$ set to be $r=0, r=0.5$ and $r=1$. The illustration of the results can be referred in Figures 11,12, and 13. It is clear that the solutions create a form of interval which indicates that the solutions are fuzzy numbers.

Table 6: Numerical solutions of $M_{1,1 \mathrm{r}}(z)$ and $M_{1,2 \mathrm{r}}(z)$ for different values of $\mathrm{r}$ and $\alpha=0.8$.

\begin{tabular}{cccccc}
\hline$z$ & $\begin{array}{c}\mathrm{M}_{1,1 \mathrm{r}}(z) \\
\mathrm{r}=0\end{array}$ & $\begin{array}{c}\mathrm{M}_{1,2 \mathrm{r}}(z) \\
\mathrm{r}=0\end{array}$ & $\begin{array}{c}\mathrm{M}_{1,1 \mathrm{r}}(z) \\
\mathrm{r}=.5\end{array}$ & $\begin{array}{c}\mathrm{M}_{1,2 \mathrm{r}}(z) \\
\mathrm{r}=.5\end{array}$ & $\begin{array}{c}\mathrm{M}_{1, \mathrm{r}}(z)=\mathrm{M}_{1,2 \mathrm{r}}(z) \\
\mathrm{r}=1\end{array}$ \\
\hline 0.0 & 2.00 & 4.00 & 2.5 & 3.5 & 3.0 \\
0.1 & 1.736841535 & 3.444681614 & 2.163801555 & 3.017721594 & 2.590761574 \\
0.2 & 1.609843308 & 3.122571631 & 1.988025389 & 2.74438955 & 2.36620747 \\
0.3 & 1.561542031 & 2.922538361 & 1.901791114 & 2.582289278 & 2.242040196 \\
0.4 & 1.573674645 & 2.810071823 & 1.88277394 & 2.500972529 & 2.191873234 \\
0.5 & 1.637606954 & 2.768810968 & 1.920407958 & 2.486009965 & 2.203208961 \\
0.6 & 1.748321 & 2.789137328 & 2.008525082 & 2.528933246 & 2.268729164 \\
0.7 & 1.902594453 & 2.864761074 & 2.143136108 & 2.624219419 & 2.383677764 \\
0.8 & 2.09823305 & 2.991302225 & 2.321500344 & 2.768034932 & 2.544767638 \\
0.9 & 2.33369741 & 3.165591107 & 2.541670834 & 2.957617683 & 2.749644258 \\
1.0 & 2.608035252 & 3.385443423 & 2.802387295 & 3.19109138 & 2.996739338 \\
\hline
\end{tabular}

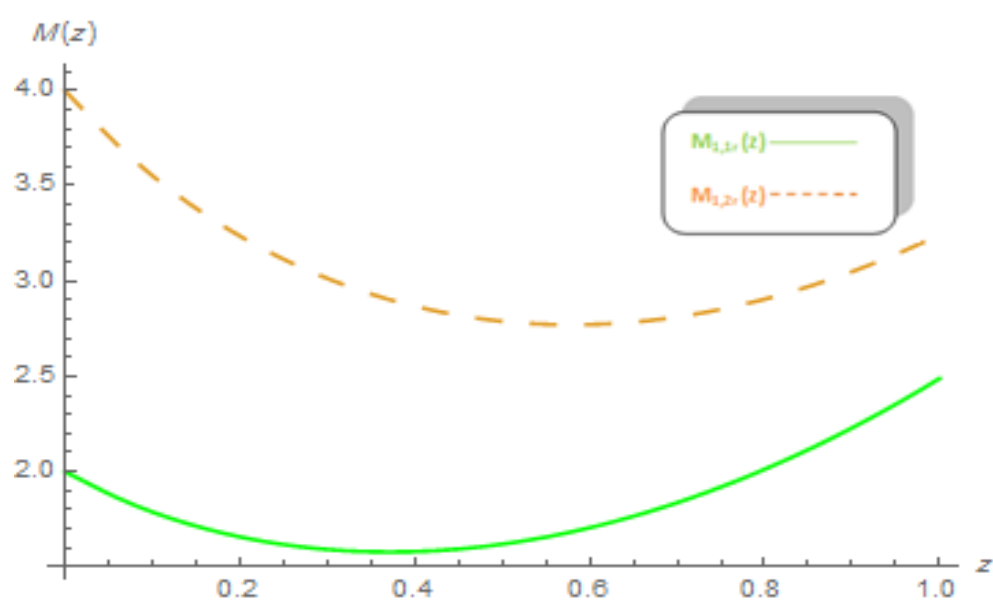

Figure 11: Approximate solutions of $M_{1,1 r}(z)$ and $M_{1,2 r}(z)$ when $r=0$ for Example 4.2. 


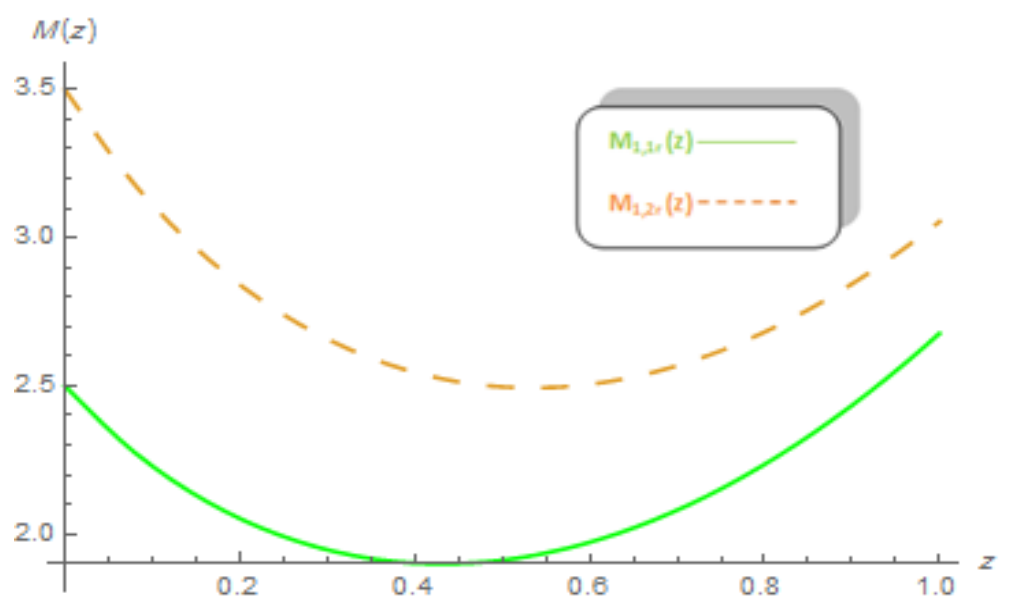

Figure 12: Approximate solutions of $M_{1,1 r}(z)$ and $M_{1,2 r}(z)$ when $r=0.5$ for Example 4.2.

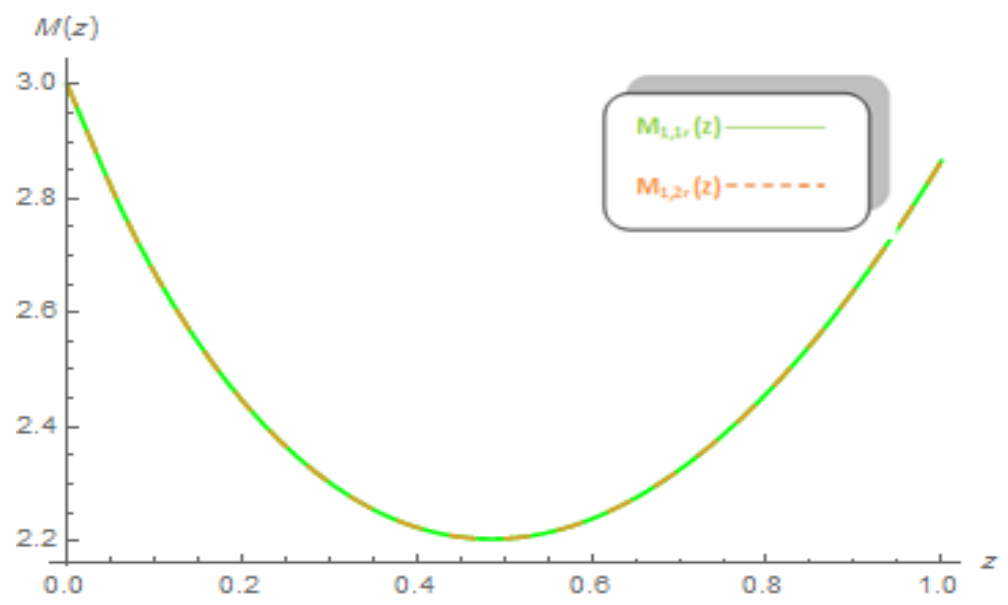

Figure 13: Approximate solutions of $M_{1,1 r}(z)$ and $M_{1,2 r}(z)$ when $r=1.0$ for Example 4.2.

\section{Conclusion}

In this paper, we have studied the solutions of fuzzy initial value problem for differential equations of integer and fractional order with Caputo derivatives. This has been done by modifying the reproducing kernel Hilbert space. N-term approximation solution is obtained from the method, and it can be further applied widely and more efficiently compared to traditional methods to solve fuzzy initial value problems for differential equations of integer order. This improved method contributes in many ways, such as solving fuzzy system effectively, reduce time for solving problems related to real phenomena and engineering applications.

\section{References}

[1] O. Abu Arqub, An iterative method for solving fourth-order boundary value problems of mixed type integro-differential equations, J. Comput. Anal. Appl., 8 (2015), 857-874. 1

[2] O. Abu Arqub, M. Al-Smadi, S. Momani, Application of reproducing kernel method for solving nonlinear FredholmVolterra integrodifferential equations, Abstr. Appl. Anal., 2012 (2012), 16 pages. 1

[3] R. P. Agarwal, V. Lakshmikantham, J. J. Nieto, On the concept of solution for fractional differential equations with uncertainty, Nonlinear Anal., 72 (2010), 2859-2862 1

[4] M. Z. Ahmad, M. K. Hasan, S. Abbasbandy, Solving fuzzy fractional differential equations using Zadeh's extension principle, Scientific World J., 2013 (2013), 11 pages. 1, 2.4 
[5] A. Ahmadian, S. Salahshour, C. S. Chan, A Runge-Kutta method with reduced number of function evaluations to solve hybrid fuzzy differential equations, Soft Comput., 19 (2015), 1051-1062. 1

[6] A. Ahmadian, N. Senu, F. Larki, S. Salahshour, A legendre approximation for solving a fuzzy fractional drug transduction model into the bloodstream, Recent Advances on Soft Computing and Data Mining, Springer International Publishing, 2014 (2014), 25-34. 1

[7] A. Ahmadian, M. Suleiman, S. Salahshour, D. Baleanu, A Jacobi operational matrix for solving a fuzzy linear fractional differential equation, Adv. Difference Equ., 2013 (2013), 29 pages.

[8] T. Allahviranloo, A. Armand, Z. Gouyandeh, Fuzzy fractional differential equations under generalized fuzzy Caputo derivative, J. Intell. Fuzzy Systems, 26 (2014), 1481-1490. 1

[9] A. K. Alomari, F. Awawdeh, N. Tahat, F. Bani Ahmad, W. Shatanawi, Multiple solutions for fractional differential equations: analytic approach, Appl. Math. Comput., 219 (2013), 8893-8903. 1

[10] D. Alpay, V. Bolotnikov, H. T. Kaptanoğlu, The Schur algorithm and reproducing kernel Hilbert spaces in the ball, Linear Algebra Appl., 342 (2002), 163-186. 1

[11] M. Al-Smadi, O. Abu Arqub, S. Momani, A computational method for two-point boundary value problems of fourth-order mixed integrodifferential equations, Math. Probl. Eng., 2013 (2013), 10 pages. 1

[12] S. Arshad, V. Lupulescu, On the fractional differential equations with uncertainty, Nonlinear Anal., 74 (2011), $3685-$ 3693. 1

[13] E. Babolian, H. Sadeghi, S. Javadi, Numerically solution of fuzzy differential equations by Adomian method, [[Numerical solution of fuzzy differential equations by the Adomian method]] Appl. Math. Comput., 149 (2004), 547-557. 2

[14] S. Bartokos, Reproducing kernel Hilbert spaces, Diplomarbeit, University of Vienna, Fakultät für Mathematik BetreuerIn: Haslinger, Friedrich, (2011). 1

[15] J. J. Buckley, E. Eslami, T. Feuring, Fuzzy mathematics in economics and engineering, Studies in Fuzziness and Soft Computing, Physica-Verlag, Heidelberg, (2002). 2

[16] J. J. Buckley, Y. Qu, Solving systems of linear fuzzy equations, Fuzzy Sets and Systems, 43 (1991), 33-43. 2

[17] Y. Chalco-Cano, H. Román-Flores, Some remarks on fuzzy differential equations via differential inclusions, Fuzzy Sets and Systems, 230 (2013), 3-20. 1

[18] M.-G. Cui, Y.-Z. Lin, Nonlinear numerical analysis in the reproducing kernel space, Nova Science Publishers, Inc., New York, (2009). 2.6, 2.7, 2.8, 2.9, 2.10, 2.11, 3

[19] V. Daftardar-Gejji, Y. Sukale, S. Bhalekar, Solving fractional delay differential equations: a new approach, Fract. Calc. Appl. Anal., 18 (2015), 400-418. 1

[20] E. C. de Oliveira, J. A. Tenreiro Machado, A review of definitions for fractional derivatives and integral, Math. Probl. Eng., 2014 (2014), 6 pages. 2.1

[21] C. Gu, Penalized likelihood estimation: convergence under incorrect model, Statist. Probab. Lett., 36 (1998), 359-364. 1

[22] J. R. Higgins, Sampling in reproducing kernel Hilbert space, New perspectives on approximation and sampling theory, Appl. Numer. Harmon. Anal., Birkhäuser/Springer, Cham, (2014), 23-38. 1

[23] H. Hult, Approximating some Volterra type stochastic integrals with applications to parameter estimation, Stochastic Process. Appl., 105 (2003), 1-32. 1

[24] M. M. Khader, Numerical treatment for solving fractional logistic differential equation, Differ. Equ. Dyn. Syst., 24 (2016), 99-107. 1

[25] A. Khastan, K. Ivaz, Numerical solution of fuzzy differential equations by Nyström method, Chaos Solitons Fractals, 41 (2009), 859-868. 2

[26] E. Khodadadi, E. Çelik, The variational iteration method for fuzzy fractional differential equations with uncertainty, Fixed Point Theory Appl., 2013 (2013), 7 pages. 1

[27] M. P. Lazarević, R. R. Milan, B. Š. Tomislav, Introduction to fractional calculus with brief historical background, Adv. Top. Appl. Fractional Calc. Control Probl. Syst. Stab. Model., 3 (2014), 82-85. 2

[28] C.-L. Li, M.-G. Cui, The exact solution for solving a class nonlinear operator equations in the reproducing kernel space, Appl. Math. Comput., 143 (2003), 393-399. 1

[29] M. Mazandarani, A.V. Kamyad, Modified fractional Euler method for solving fuzzy fractional initial value problem, Commun. Nonlinear Sci. Numer. Simul., 18 (2013) , 12-21. 1

[30] J. Mercer, Functions of positive and negative type, and their connection with the theory of integral equations, Trans. London Philosophical Soc. A, 209 (1909), 415-446. 1

[31] O. H. Mohammed, F. S. Fadhel, F. A. Abdul-Khaleq, Differential transform method for solving fuzzy fractional initial value problems, J. Basrah Res., 37 (2011), 158-170. 1

[32] S. Momani, Z. Odibat, Numerical approach to differential equations of fractional order, J. Comput. Appl. Math., 207 (2007), 96-110. 2.2

[33] A. Salah, M. Khan, M. A. Gondal, A novel solution procedure for fuzzy fractional heat equations by homotopy analysis transform method, Neural Comput. Appl., 23 (2013), 269-271. 1

[34] S. Salahshour, T. Allahviranloo, Application of fuzzy differential transform method for solving fuzzy Volterra integral equations, Appl. Math. Model., 37 (2013), 1016-1027. 1

[35] S. Salahshour, T. Allahviranloo, S. Abbasbandy, Solving fuzzy fractional differential equations by fuzzy Laplace transforms, Commun. Nonlinear Sci. Numer. Simul., 17 (2012), 1372-1381.1 
[36] D. J. Strauss, G. Steidl, Hybrid wavelet-support vector classification of waveforms, J. Comput. Appl. Math., 148 (2002), 375-400. 1

[37] L.-H. Yang, M.-G. Cui, New algorithm for a class of nonlinear integro-differential equations in the reproducing kernel space, Appl. Math. Comput., 174 (2006), 942-960. 1

[38] K. Yao, X.-W. Chen, A numerical method for solving uncertain differential equations, J. Intell. Fuzzy Systems, 25 (2013), 825-832. 1

[39] Y. Zhang, G.-Y. Wang, Time domain methods for the solutions of n-order fuzzy differential equations, Fuzzy Sets and Systems, 94 (1998), 77-92. 2 